\title{
ANÁLISE MORFOLÓGICA DOS PROCESSOS PATOLÓGICOS GERAIS EM RINS DE RATOS OBESOS E SUA RELAÇÃO COM O EXERCÍCIO FÍSICO
}

Lucas Freitas Bergamaschi Pereira da Silva ${ }^{1}$, Rebeca Carnavale Bequer ${ }^{1}$, Fabíola de Azevedo de Mello ${ }^{1}$, Marcela de Andrade Bernal Fagiani ${ }^{1}$, Ana Karina Marques Salge ${ }^{2}$, Débora Tavares de Resende e Silva ${ }^{3}$, Lucas Agostini ${ }^{4}$, Thaís Batista Dutra ${ }^{4}$, Armando Florido Neto ${ }^{4}$, Patricia Monteiro Seraphim ${ }^{4}$ Thiago Milani da Costa ${ }^{1}$, Abel Gomes de Pinheiro Neto ${ }^{1}$, Bruna Maria Casachi Bernardes de Melo Carapeba ${ }^{1}$, Renata Calciolari Rossi ${ }^{1}$

${ }^{1}$ Universidade do Oeste Paulista - UNOESTE, Faculdade de Medicina, Presidente Prudente, SP. ${ }^{2}$ Universidade Federal de Goiás - UFG, Goiânia, GO. ${ }^{3}$ Universidade Federal Fronteira do Sul - UFFS, Chapecó, SC. ${ }^{4}$ Faculdade de Ciências e Tecnologia de Presidente Prudente - FCT-UNESP, Presidente Prudente, SP. e-mail: fabiola-azevedo@hotmail.com

\section{RESUMO}

O objetivo do estudo foi comparar os achados morfológicos, quantificar a inflamação e fibrose renal em ratos obesos submetidos ao exercício físico. Foram utilizados 56 ratos machos Wistar. Os animais controles foram alimentados com ração padrão e água de torneira, e os obesos por uma dieta hiperlipídica. Esses animais foram submetidos ao treinamento intermitente, onde se realizou o movimento de extensão completa da pata, levantando uma carga posicionada na parte posterior do colete. Foram coletados os rins dos animais para avaliar os achados. Com relação à análise histopatológica, não foram evidenciados processos patológicos gerais. Ao ser realizada a quantificação de fibrose, não foram evidenciados resultados significativos entre os grupos. Conclui-se que não foram evidenciados processos patológicos gerais em rins de ratos obesos e sua relação com o exercício físico. Desta forma, é sugerida a realização de novos estudos com protocolos mais longos a fim de elucidar melhor os resultados.

Palavras-chaves: Obesidade, rim, Inflamação, fibrose, exercício.

\section{MORPHOLOGICAL ANALYSIS OF GENERAL PATHOLOGICAL PROCESSES IN KIDNEYS OF OBESE RATS AND THEIR RELATIONSHIP WITH PHYSICAL EXERCISE}

\begin{abstract}
The objective of the study was to compare the morphological findings, quantify the inflammation and renal fibrosis in obese rats submitted to physical exercise. Male Wistar rats were used. Control animals were fed standard feed and tap water, and the obese were fed a hyperlipidic diet. These animals were submitted to intermittent training, where the movement of complete extension of the paw was performed, lifting a load positioned at the back of the vest. The animals' kidneys were collected to evaluate the findings. Regarding the histopathological analysis, no general pathological processes were evidenced. When the fibrosis quantification was performed, no significant results were observed between the groups. It was concluded that no general pathological processes were observed in the kidneys of obese rats and their relationship with physical exercise. In this way, it is suggested to carry out new studies with longer protocols in order to better elucidate the results.
\end{abstract}

Keywords: Obesity, kidney, inflammation, fibrosis, exercise. 


\section{INTRODUÇÃO}

A obesidade é caracterizada pelo acúmulo de gordura corporal na forma de adiposo $^{1}$ por consequência do excesso de consumo de alimentos calóricos associados ao sedentarismo. Nas últimas décadas, a prevalência da obesidade tem aumentado em proporções alarmantes no mundo inteiro ${ }^{2}$. Obesidade e doença renal são motivos de preocupação mundial e sério problema na saúde pública, um exemplo é os Estados Unidos onde $60 \%$ das mulheres e $51 \%$ dos homens estão classificados com sobrepeso ou obesos devido ao aumento intenso do número de casos nos últimos 20 anos $^{3}$.

Estudos têm demonstrado a relação da obesidade com a doença renal crônica através de um mecanismo multifatorial. A presença da obesidade no começo da vida está ligada à baixa taxa de filtração glomerular e o sobrepeso na idade adulta dobra as chances de doença renal crônica ${ }^{4}$.

O rim é um órgão complexo cuja função principal é atuar como filtro incansável transformando cerca de 1700 litros de sangue em 1 litro de urina a cada dia, concomitante a eliminação de toxinas e escórias metabólicas; com papel essencial na homeostase do organismo ${ }^{5}$. Os rins apresentam formato oval, coloração marrom-avermelhada e medem cerca de $10 \mathrm{~cm}$ de comprimento, $5 \mathrm{~cm}$ de largura e 2,5 $\mathrm{cm}$ de espessura. Superiormente, estão associados ao diafragma; inferiormente, suas faces posteriores estão associados aos músculos psoas maior medialmente e quadrado do lombo. O rim direito se relaciona com o fígado, duodeno e colo ascendente e o rim esquerdo se relaciona com estômago, baço, pâncreas, jejuno e colo descendente ${ }^{6}$.

A obesidade é considerada um fator independente da doença renal crônica, segundo estudos, além de estar associada a taxas de morbidade mais altas e a doenças relacionadas à obesidade. Existe a atuação de diversas enzimas como a leptina e a adiponectina, citocinas como TNF- $\alpha$, IL-6 e MCP-1 e infiltrado celular com perfil pró-inflamatório na ocorrência da doença renal crônica ${ }^{4}$. O rápido ganho de peso causa inicialmente uma reabsorção tubular renal de sódio aumentada que leva à vasodilatação renal compensatória e taxa de filtração glomerular (TFG) aumentada. Os mecanismos compensatórios também levam ao aumento da tensão da parede glomerular e hipertrofia glomerular, mudanças que podem levar à lesão renal, glomeruloesclerose e perda renal ${ }^{7}$.

$\mathrm{Na}$ doença renal crônica, estudos comprovam a importância da inflamação na fisiopatologia. Essa inflamação está associada à elevação de níveis séricos de proteínas inflamatórias de fase aguda e uma variedade de mediadores imuno-inflamatórios sendo que o último modula a função endotelial, adesão e migração das células circulantes do sistema imune para o interstício e apresentam capacidade de ativar fibroblastos residentes ${ }^{8}$.

A atividade física regular em pacientes renais crônicos possui uma grande importância por apresentar um efeito anti-inflamatório através da redução dos níveis séricos de componentes inflamatórios comumente presentes nesses pacientes como a IL-6 e PCR ${ }^{9}$.

Devido à grande prevalência da obesidade na população mundial conjuntamente com o aumento do número de novos casos de obesos e sobrepesos e ao grande número de casos de doença renal crônica associada também à diálise em seu grau de comprometimento renal, uma pesquisa mais incisiva sobre a correlação entre a obesidade e a doença renal será de grande elucidação para a área da saúde.

A hipótese é de que ratos obesos e sedentários apresentem mais inflamação e fibrose no parênquima renal quando comparados com aqueles que realizaram exercícios físicos ${ }^{10}$. 0 objetivo do estudo foi comparar os achados morfológicos, quantificar a inflamação e fibrose renal em ratos obesos submetidos ao exercício físico.

\section{METODOLOGIA}

O projeto foi executado após aprovação do comitê de ética em animais da Universidade do Oeste Paulista (protocolo 2534). Os procedimentos relacionados aos protocolos de experimentação (exposição à fumaça do cigarro e ao álcool) até a eutanásia dos animais foram executados na Faculdade de Ciências e Tecnologia de Presidente Prudente e aprovado sob o processo no 2/2013.

\section{Procedimento Experimental}

Junto ao Biotério central, foram obtidos 56 ratos machos Wistar pesando em torno de 200g e permaneceram no biotério para animais da Faculdade de Ciências e Tecnologia UNESP campus de Presidente Prudente, em gaiolas plásticas $(30 \times 16 \times 19 \mathrm{~cm})$ coletivas. Esses ratos 
machos Wistar permaneceram em grupos de 3 a 5 animais por gaiola, no biotério, sob temperatura média de $22 \pm 2$ e ciclo claro/escuro de 12 horas, sendo o ciclo claro iniciado as 7:00 horas. Os animais controles foram alimentados com ração padrão e água de torneira fornecida ad libitum, já os grupos obesos seguiram uma dieta hiperlipídica (dieta de cafeteria) ${ }^{11}$, composta por mortadela, bacon, salsicha, bolacha, refrigerante e ração padrão, numa proporção de aproximadamente 2:2:2:1:1:1 respectivamente, numa composição de $28 \%$ de carboidratos, $13 \%$ de proteínas e $59 \%$ de lipídeos a partir do 20 mês de vida. O peso corpóreo (PC) dos ratos machos Wistar foi registrado semanalmente.

Os animais foram divididos em quatro grupos: Exercício (E), Obeso Exercício (OE), Sedentário (S) e Obeso Sedentário (OS).

\section{Treinamento Intermitente}

Com algumas adaptações, foi utilizado o modelo de treinamento intermitente ${ }^{12}$. O aparelho foi projetado de modo que o rato macho Wistar ficasse imobilizado por um colete adaptado sobre uma plataforma metálica.

Com auxilio de um clipe metálico, foram realizadas estimulações elétricas a extremidade da cauda do animal ligado a um eletroestimulador do tipo Dualpex 961, da Quarker, calibrado pelo INMETRO. Os parâmetros utilizados foram: frequência 1 Herts $(\mathrm{Hz})$, duração de 0,3 segundos com intervalo de 2 segundos entre cada estimulação elétrica e, a intensidade ajustada de maneira que o animal executou o movimento, variando de 3 a 6 miliamperes (mA). Esses parâmetros foram adotados, pois são pulsos bidirecionais de média nula, não se manifestando efeitos eletrolíticos e permitindo aplicações de longa duração sem risco de lesão dos tecidos.

O rato macho Wistar realizou a partir dessa estimulação o movimento de extensão completa da pata (joelho e tornozelo) levantando uma carga posicionada na parte posterior do colete. O protocolo de treinamento será de 3 séries de 12 repetições, 3 vezes por semana durante 8 semanas. Na primeira semana foram realizadas três sessões de adaptação sem incremento de carga: 1,2 e 3 séries de 2 repetições do primeiro ao terceiro dia respectivamente. Nas primeiras duas semanas, os animais realizaram o treinamento sem incremento de carga. Nas terceira e quarta semana foram impostas uma carga equivalente a $50 \%$ do peso corporal $^{13}$. No período correspondente a quinta e sexta semana, a carga foi ajustada a $60 \%$ do peso corporal do animal. Durante há sétima semana a carga utilizada era de $70 \%$ do peso corpóreo do animal, seguindo assim até o fim do protocolo de treinamento. A carga utilizada para o teste agudo foi $50 \%$ do peso corporal do animal.

De acordo com as variações da massa corporal, a carga semanalmente foi ajustada. O movimento de "squat jump" foi testado e avaliado como indutor de hipertrofia em animais semelhante à hipertrofia obtida em humanos levantadores de peso ${ }^{14}$.

$\mathrm{O}$ treinamento se iniciou juntamente com a dieta lipídica, quando os animais atingiram idade jovem, adulta de 2 meses de idade.

Após a fase de treinamento, os animais foram eutanasiados com um corte na artéria aorta após sedação por anestésicos Quetamina e Xilazina (40 mg/Kg do peso corporal) por via intraperitoneal. Posteriormente, foi realizada autópsia dos animais para retirada dos rins para análise histopatológica.

\section{Avaliação Histológica}

Após fixação em Boin, foram coletados fragmentos da região central do rim esquerdo e emblocados em parafina. Foram confeccionadas lâminas histológicas com a espessura de $5 \mu \mathrm{m}$.

Técnicas de Coloração

Hematoxilina Eosina

É a mais adequada técnica para a análise de células inflamatórias, onde a hematoxilina foi dissolvida no álcool e o alúmen na água destilada (previamente aquecida). Posteriormente as duas soluções foram misturadas e aquecidas até a fervura. O óxido de mercúrio foi adicionado à solução resfriada, mergulhando-se o frasco em água fria. $O$ ácido acético foi então colocado na solução fria para finalmente ser filtrada. Esse processo durou em torno de 5 a $15 \mathrm{~min}$, e após a lavagem do material em água corrente, e passagem rápida em álcool $95^{\circ}$, foi aplicada a coloração em Eosina, e posteriormente diferenciada em álcool $95^{\circ}$.

\section{Tricrômio de Masson}

Os cortes histológicos foram desparafinizados com xilol e passados em álcool puro. Foram mergulhados em solução de Alumen de amônio-férrrico a $45-50^{\circ} \mathrm{C}$ na estufa por $5 \mathrm{~min}$. 
Posteriormente foram corados pela hematoxilina de Regaud aquecida a 45 a $50^{\circ} \mathrm{C}$ na estufa por 5 min. Foi utilizado o diferenciador no álcoolpícrico ( $3 \mathrm{~min}$ ). Após as lavagens com água corrente foram corados na $A^{*}$ por 5 min, $B^{* *}$ por mais 5 min e gotejamento de $C^{* * *}$ por mais 5 min. Finalmente foram mergulhados em solução aquosa de ácido acético a 1\% por 5 min.

Análise de resultados

Para a análise dos resultados foi confeccionado um banco de dados eletrônico. Foi realizada a análise de variância ANOVA nos casos de distribuição paramétrica, seguido pelo teste de Tukey quando necessário e Kruskall-Wallis, seguido pelo teste de Dunn nos casos de distribuição não paramétrica. Foram significativos os valores de $p<0,05$.

\section{RESULTADOS}

Foram analisados 40 animais submetidos à dieta hipercalórica e exercício físico. Em relação ao peso dos animais, foi verificado que o grupo obeso sedentário apresentou aumento significativo no peso pós-intervenção quando comparado com o grupo pré-intervenção, associado também ao menor desvio padrão $(p=0,007)($ Tabela 1$)$.
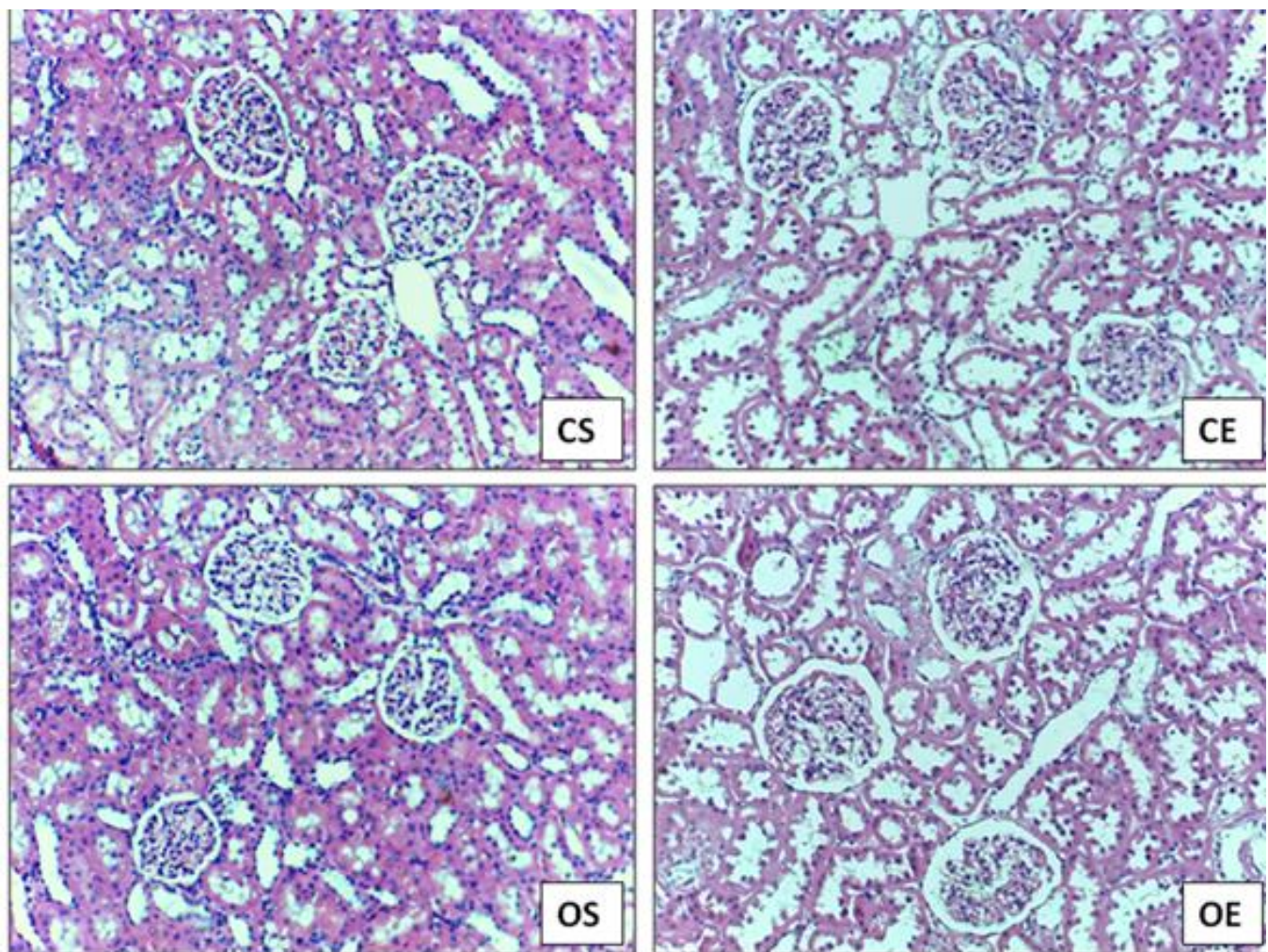

Figura 1. Análise dos processos patológicos gerais em rins de ratos obesos e sua relação com o exercício físico. Exercício (E), Obeso Exercício (OE), Sedentário (S) e Obeso Sedentário (OS). 

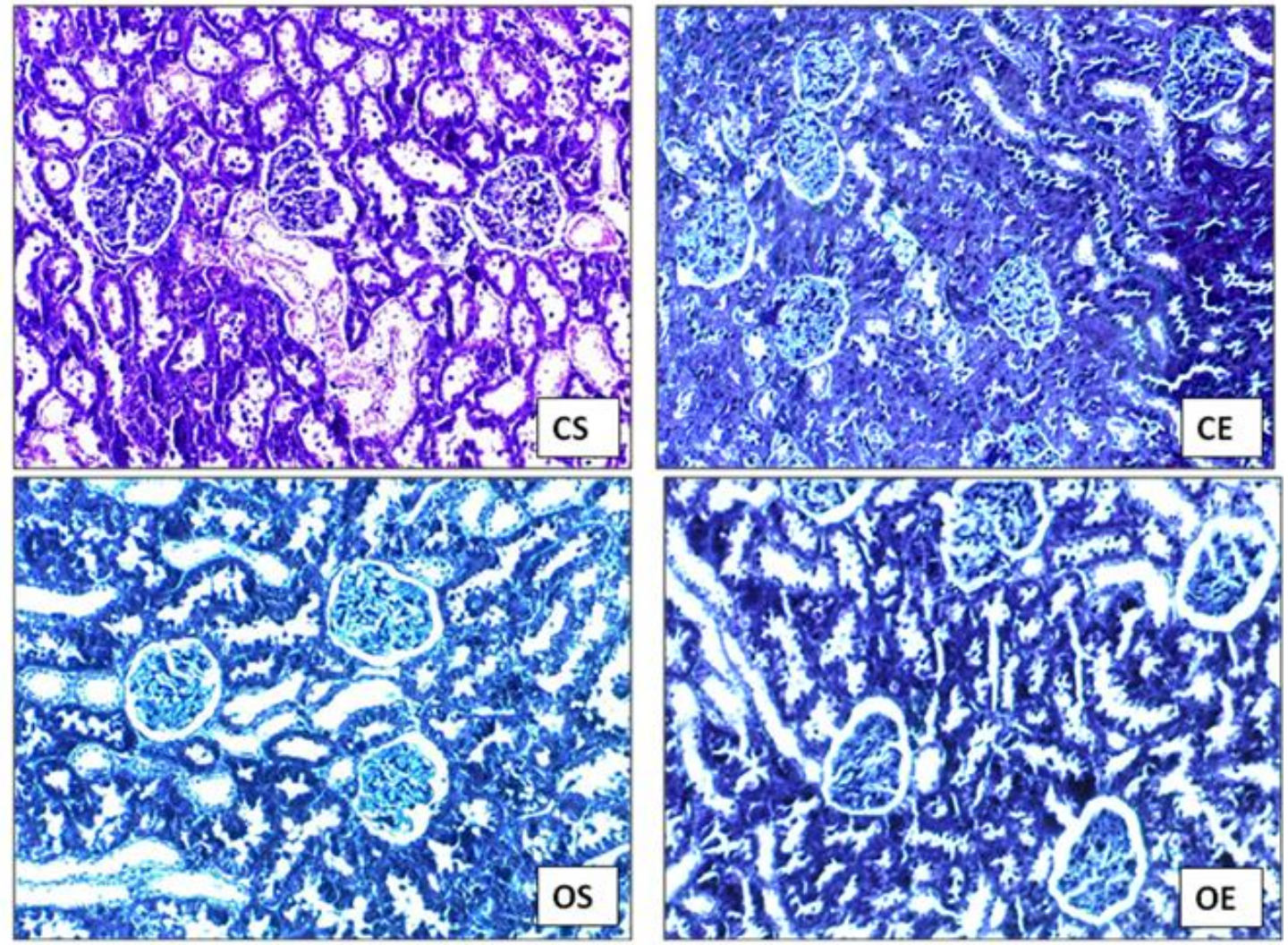

Figura 2. Quantificação de fibrose em rins de ratos obesos submetidos ao treinamento físico. Exercício (E), Obeso Exercício (OE), Sedentário (S) e Obeso Sedentário (OS).

Tabela 2. Quantificação de fibrose em rim de ratos obesos submetidos ao exercício físico.

\begin{tabular}{lcc}
\hline Grupos & $\mathrm{n}(\%)$ & $\mathrm{Med}(25 \%-75 \%)$ \\
\hline Controle sedentário & $10(25)$ & $0,8(0,5-1,1)$ \\
Controle exercício & $10(25)$ & $0,9(0,4-1,5)$ \\
Obeso exercício & $10(25)$ & $0,8(0,5-1,3)$ \\
Obeso sedentário & $10(25)$ & $0,8(0,6-1,1)$ \\
Total & $40(100)$ & \\
\hline
\end{tabular}

$\mathrm{p}=0,295$; Teste de Kruskal-Wallis.

\section{DISCUSSÃO}

O exercício representa um estresse físico que desafia a homeostase. O desafio inclui a clínica, o paciente, fatores sistêmicos e o ambiente que envolve o paciente nos quesitos psicológico, bioquímico e parâmetros imunológicos ${ }^{15}$.

A literatura mostra que existe uma correlação entre o exercício físico e a piora da morfologia renal representado pelo aumento de áreas mesangiais, aumento das concentrações plasmáticas de ureia e de creatina quinase (CK) acarretando na piora do perfil morfológico renal $^{16}$. Em nosso estudo não encontramos resultados significativos com relação à morfologia renal. Tal dado pode estar relacionado ao tempo de protocolo de treinamento físico.

Analisando o trabalho realizado por Chiung-Chi et al. ${ }^{16}$, observa-se 0 efeito do exercício físico sobre o rim, função renal e o ganho de peso, pode melhorar os quadros de edema renal, em relação à deposição de colágeno e diminuição da produção de citocinas inflamatórias. Em nosso estudo observamos que somente os pesos dos animais apresentaram resultados significativos. Uma das limitações deste estudo foi a não realização de exames laboratoriais e bioquímicos. Acreditamos que talvez os resultados oriundos de perfis hematológicos pudessem apresentar resultados mais sensíveis de ser observados.

\section{CONCLUSÃO}

Não foram encontrados processos patológicos gerais em rins de ratos obesos e sua relação com o exercício físico. Desta forma é sugerida a realização de novos estudos com protocolos mais longos a fim de elucidar melhor os resultados. 


\section{DECLARAÇÃO DE CONFLITO DE INTERESSE}

Os autores declaram não haver qualquer potencial conflito de interesse que possa interferir na imparcialidade deste trabalho científico.

\section{REFERÊNCIAS}

1. Bergamaschi CT, Boim MA, Moura LA, Piçarro IC, Schor N. Effects of long-term training on the progression of chronic renal failure in rats. Med Sci Sports Exerc. 1997;29:169-74. DOI: https://doi.org/10.1097/00005768-199702000$\underline{00001}$

2. Halpern A. A epidemia de obesidade. Arq Bras Endocrinol Metab. 1999;43(3):175-6. DOI: https://doi.org/10.1590/S0004-

27301999000300002

3. Cecil RL, Goldman L, Ausiello D. Cecil Medicina. 24.ed. Rio de Janeiro: Editora Saunders Elsevier, 2014. v.1. p.927-1618.

4. Felizardo RJF, da Silva MB, Aguiar CF, Câmara NOS. Obesity in kidney disease: a heavyweight opponent. World J Nephrol. 2014;3(3):50-63. DOI: http://dx.doi.org/10.5527/win.v3.i3.50

5. Filho GB. Bogliolo Patologia. 8.ed. Rio de Janeiro: Guanabara Koogan; 2011. 519p.

6. Moore KL, Dalley AF, Agur AMR. Anatomia Orientada para a clínica. 6.ed. Rio de Janeiro: Guanabara Koogan; 2011. p.290-1.

7. Hall ME, Carmo JM, Silva AA, Juncos LA, Wang Z, Hall JE. Obesity, hypertension, and chronic kidney disease. Int $\mathrm{J}$ Nephrol Renovasc Dis. 2014;18(7):75-88.

DOI:

http://dx.doi.org/10.2147/IJNRD.S3973

8. Vianna HR, Soares CMBM, Tavares MS, Teixeira $M M$, Silva ACS. Inflamação na doença renal crônica: papel de citocinas. J Bras Nefrol. 2011;33(3):351-64.

DOI:

https://doi.org/10.1590/s0101-

$\underline{28002011000300012}$

9. Dungey M, Hull KL, Smith AC, Burton JO, Bishop NC. Inflammatory factors and exercise in chronic kidney disease. Int J Endocrinol. 2013:569831. DOI: https://doi.org/10.1155/2013/569831
10. Tirapegui J. Atividade física e obesidade. Rev Bras Cienc Farm. 2003;39(4):467. DOI: https://doi.org/10.1590/S1516-

$\underline{93322003000400015}$

11. Panveloski-Costa $A C$, Pinto Júnior DAC, Brandão BB, Moreira RJ, Machado UF, Seraphim $P M$. Treinamento resistido reduz inflamação em músculo esquelético e melhora a sensibilidade à insulina periférica em ratos obesos induzidos por dieta hiperlipídica. Arq Bras Endocrinol Metab. 2011;55(2):155-163.

DOI: https://doi.org/10.1590/S0004$\underline{27302011000200008}$

12. Tamaki T, Uchiyama S, Nakano S. A weightlifting exercise model for inducing hypertrophy in the hindlimb muscles of rats. Med Sci Sports Exerc. 1992;24(8):881-6. DOI: https://doi.org/10.1249/00005768-199208000$\underline{00009}$

13. Barbosa NO, Abate DT, Marocolo Júnior $M$, Mota GR, Orsatti FL, Rossi e Silva RC, Reis MA, Silva VJ. Exercise training improves cardiovascular autonomic activity and attenuates renal damage in spontaneously hypertensive rats. J Sports Sci Med. 2013;12(1):52-59.

14. Vera-Cruz $M$, Nunes $M$, Mendonça $L$, Chaves E, Fernandes MLLA. Efeito do chá verde (Camelia sinensis) em ratos com obesidade induzida por dieta hipercalórica. J Bras Patol Med Lab. 2008;46(5):407-413. DOI: https://doi.org/10.1590/S1676$\underline{24442010000500010}$

15. Aparicio VA, Tassi $M$, Nebot E, CamilettiMoirón D, Ortega E, Porres JM, Aranda P. Highintensity exercise may compromise renal morphology in rats. Int J Sports Med. 2014;35(8):639-44. DOI: http://dx.doi.org/10.1055/s-0033-1354383

16. Chiung-Chi P, Kuan-Chou C, Chiu-Lan H, Robert YP. Swimming exercise prevents fibrogenesis in chronic kidney disease by inhibiting the myofibroblast trans differentiation. PLOS One. 2012;7(6):1-17. DOI: https://doi.org/10.1371/journal.pone.0037388 